\title{
Performing forward-viewing endoscopy at time of pancreaticobiliary EUS and ERCP may detect additional upper gastrointestinal lesions
}

Authors

Institutions
Ashby Thomas ${ }^{1,}$ * , Arunan S Vamadevan ${ }^{1, *}$, Eoin Slattery ${ }^{2}$, Divyesh V Sejpal' , Arvind J Trindade ${ }^{1}$

${ }^{1}$ Division of Gastroenterology, Department of Medicine, Hofstra North Shore Long Island Jewish School of Medicine, Long Island Jewish Medical Center, New Hyde Park, NY

${ }^{2}$ Department of Preventive Medicine and Nutrition, Columbia University Medical Center, New York, NY submitted:

17. July 2015

accepted after revision:

4. November 2015

\section{Bibliography}

DOI http://dx.doi.org/

10.1055/s-0041-109084

Published online: 11.1.2016

Endoscopy International Open

2016; 04: E193-E197

(c) Georg Thieme Verlag KG

Stuttgart $\cdot$ New York

E-ISSN 2196-9736

\section{Corresponding author}

Arvind J. Trindade, MD

Long Island Jewish Medical Center

Division of Gastroenterology 270-05 76th Avenue

New Hyde Park NY 11040

Phone: +1-718-470-7281

Fax: +1-718-343-0128

arvind.trindade@gmail.com
Background and study aims: It is unknown whether significant incidental upper gastrointestinal lesions are missed when using non-forwardviewing endoscopes without completing a forward-viewing exam in linear endoscopic ultrasound (EUS) or endoscopic retrograde cholangiopancreatography (ERCP) exams. We evaluated whether significant upper GI lesions are missed during EUS and ERCP when upper endoscopy is not performed routinely with a gastroscope.

Patients and methods: A retrospective analysis was performed in which an EGD with a forwardviewing gastroscope was performed after using a non-forward-viewing endoscope (linear echoendoscope, duodenoscope, or both) during a single procedure. Upper gastrointestinal tract findings were recorded separately for each procedure. Significant lesions found with a forward-viewing gastroscope were defined as findings that led to a change in the patient's medication regimen, additional endoscopic surveillance/interventions, or the need for other imaging studies.

\section{Introduction \\ $\nabla$}

Endoscopic retrograde cholangiopancreatography (ERCP) and endoscopic ultrasound (EUS) are increasingly utilized in the field of gastroenterology to diagnose and treat various upper gastrointestinal diseases. More than 500,000 ERCPs are performed annually in the United States for pancreaticobiliary disorders and the role of EUS has continued to expand over the past three decades to include a wide range of diagnostic and therapeutic interventions $[1,2]$.

Non-forward-viewing ERCP duodenoscopes and EUS linear echoendoscopes are utilized in the upper gastrointestinal tract to perform various diagnostic and therapeutic maneuvers. Their endo-
Results: A total of 168 patients were evaluated. In 83 patients, a linear echoendoscope was used, in 52 patients a duodenoscope was used, and in 33 patients both devices were used. Clinically significant additional lesions diagnosed with a gastroscope but missed by a non-forward-viewing endoscope were found in $30 / 168$ patients (18\%). EGD after linear EUS resulted in additional lesion findings in $17 / 83$ patients $\left(20.5 \%, X^{2}=13.385, P=\right.$ 0.00025). EGD after use of a duodenoscope resulted in additional lesions findings in $10 / 52 \mathrm{pa}-$ tients $\left(19.2 \%, X^{2}=9.987, P=0.00157\right)$. EGD after the use of both a linear echoendoscope and a duodenoscope resulted in additional lesions findings in $3 / 33$ patients $\left(9 \%, X^{2}=3.219, P=0.07\right)$.

Conclusion: Non forward-viewing endoscopes miss a significant amount of incidental upper gastrointestinal lesions during pancreaticobiliary endoscopy. Performing an EGD with a gastroscope at the time of linear EUS or ERCP can lead to increased yield of upper gastrointestinal lesions.

scopic fields of view limit visualization of the lumen compared to the standard upper endoscope because these endoscopes are not designed to diagnose upper gastrointestinal tract lesions. Duodenoscopes and linear echoendoscopes are sideviewing and oblique-viewing endoscopes, respectively, with a field of view of 100 degrees, as compared to the 140-degree field of view of the standard gastroscope ( Table1). In addition, these non-forward-viewing endoscopes have a larger diameter and more rigid distal tip, limiting their maneuverability and angulation range. While these scopes are used for specific indications, the examination of the upper gastrointestinal tract is limited and luminal lesions may be missed. A recent study evaluating the utility of standard upper endoscopy before EUS demonstrated that clinically meaningful lesions were found on esophagogastroduodenoscopy (EGD) in 


\begin{tabular}{|llll|}
\hline & EUS Linear Endoscope & ERCP duodenoscope & Gastroscope \\
\hline Field of view & $100^{\circ}$ & $100^{\circ}$ & $140^{\circ}$ \\
\hline Direction of view & forward, oblique-viewing & & \\
\hline $\begin{array}{l}\text { Angulation range } \\
\text { (degree) }\end{array}$ & $\begin{array}{l}\text { up } 130^{\circ}, \text { down } 90^{\circ} \text {, right } \\
\text { backward, side-viewing } 5^{\circ}\end{array}$ & $\begin{array}{l}\text { up } 120^{\circ} \text {, down } 90^{\circ}, \\
\text { right } 110^{\circ} \text {, left } 90^{\circ}\end{array}$ & $\begin{array}{l}\text { forward-viewing } 210^{\circ} \text {, down } 90^{\circ} \text {, right } \\
100^{\circ} \text {, left } 100^{\circ}\end{array}$ \\
\hline
\end{tabular}

Table 1 Characteristics of the endoscopes used in the study.

EUS, endoscopic ultrasound; ERCP, endoscopic retrograde cholangiopancreatography

$22 \%$ of patients [3]. This study shows that a substantial amount of patients undergoing EUS have significant incidental luminal lesions. However, this study did not assess whether non-forwardviewing echoendoscopes would have missed meaningful lesions without the use of a gastroscope.

Although an increasing number of ERCP and EUS procedures are being performed, there is currently no consensus on the use of a standard gastroscope in tandem with these procedures. Thus, the prevalence of missed luminal lesions when only using a non-forward-viewing endoscope is unclear. The purpose of the current study was to examine the prevalence of clinically significant incidental upper gastrointestinal tract lesions that were found using a standard forward-viewing gastroscope following an ERCP or EUS exam using a duodenoscope or linear echoendoscope. We hypothesized prior to analyzing the data that linear EUS and ERCP would miss significant esophageal gastrointestinal lesions but not gastric or duodenal lesions. This was hypothesized because very little esophageal mucosa is seen while traversing the esophagus with a linear echoendoscope or duodenoscope.

\section{Patients and methods}

$\nabla$

This is a retrospective analysis of a single therapeutic endoscopist's procedures from $10 / 1 / 2013$ to $6 / 1 / 2014$ at a tertiary care medical center. After two missed gastric adenocarcinomas were presented at the hospital tumor board in patients who had previously undergone ERCPs by another gastroenterologist, the institution adopted as standard of care routine EGD at the time of all ERCP or EUS examinations. The endoscopist chose to perform the ERCP or EUS first before the EGD because his previous standard of care was to perform linear EUS or ERCP without a formal EGD if the patient had no upper gastrointestinal tract symptoms and, in the event that patient tolerability of the procedure was not ideal and early termination of the procedure was required, the maximum time would be allotted to the main EUS or ERCP procedure.

Patients were included in this study if they: 1) underwent ERCP and/or EUS using a duodenoscope (TJF-Q180V, Olympus America, Center Valley, PA, USA) or a curved linear echoendoscope (GFUCT180, Olympus America, Center Valley, PA, USA) followed by an EGD using a standard forward-viewing gastroscope (HQ190, Olympus America, Center Valley, PA, USA) during a single procedure; and 2) did not have dysphagia or other upper gastrointestinal tract symptoms. All patients were screened for dysphagia prior to undergoing the procedure. All patients signed informed consent for ERCP and/or EUS and EGD prior to the procedure. All patients had undergone monitored anesthesia care with propofol sedation or general anesthesia. Patients were excluded from this analysis if: 1) they required an EGD for diagnostic purposes; 2) they had dysphagia; 3 ) there was a clinical suspicion of upper gastrointestinal tract lesions; 4) EUS was performed using a ra- dial echoendoscope; 5) EUS exam was not a pancreaticobiliary exam; and 6) they had altered pancreaticobiliary anatomy.

All endoscopy reports recorded endoscopic lesions detected in the upper gastrointestinal tract. The data collected included age, gender, ethnicity, exam type, and indication for exam. The oblique-viewing linear echoendoscope and side-viewing duodenoscope were passed in the usual fashion for the relevant EUS and ERCP exams. Upper gastrointestinal luminal findings visualized during passage of the scopes were recorded separately for each procedure. Relevant histologic findings were recorded and correlated with endoscopic findings.

The primary outcome of the study was to determine the proportion of clinically significant missed incidental lesions when using a side- or oblique-viewing endoscope as compared to the standard forward-viewing endoscope. Significant additional lesions found with a forward-viewing gastroscope were defined as findings that led to a change in the patient's medication regimen, additional endoscopic surveillance or interventions, or the need for other imaging studies. Gastric erythema, Helicobacter pylori-negative gastritis, hiatal hernias or other anatomic abnormalities that did not affect management were not considered significant findings. Data analysis was performed using SAS 9.2 (SAS Institute Inc., Cary, NC, USA). Differences between groups were determined by using the Student's $T$ test or Wilcoxon test for continuous variables and the Fisher exact test for categorical variables. The retrospective study was approved by the Hofstra North Shore Long Island Jewish School of Medicine institutional review board.

\section{Results}

$\nabla$

A total of 168 patients were identified who underwent ERCP and/ or EUS using a side- or oblique-viewing endoscope followed by an EGD using a forward-viewing gastroscope during a single procedure. The baseline patient demographics and indication for initial procedures are included in Table2. Sixty-eight men (40\%) and 100 women (60\%) were identified. The median patient age was 55 years (range: 19-92). The most common indications for the initial procedure were known or suspected choledocholithiasis, pancreatic mass, chronic pancreatitis, or evaluation of dilated pancreatic or bile ducts.

Of the 168 patients, 52 procedures were done with a duodenoscope followed by a gastroscope, 83 procedures were done with a curved linear echoendoscope followed by a gastroscope, and 33 patients were done using both a linear echoendoscope and a duodenoscope followed by a gastroscope. Of the latter patients, $14(8 \%)$ had significant incidental clinical lesions found using a non-forward-viewing endoscope, including gastric/duodenal ulcers, $H$. pylori gastritis, duodenal polyp, and an ampullary adenoma, all of which were not included in the calculations for lesions missed by non-forward-viewing endoscopes.

Clinically significant additional lesions diagnosed with a gastroscope but missed by a non-forward-viewing endoscope were 
Table 2 Patient demographics and indications for each procedure.

\begin{tabular}{|c|c|}
\hline \multicolumn{2}{|l|}{ Patient Demographics } \\
\hline \multicolumn{2}{|l|}{ Age (years) } \\
\hline Mean & 57 \\
\hline Median & 55 \\
\hline Minimum & 19 \\
\hline Maximum & 92 \\
\hline \multicolumn{2}{|l|}{ Gender } \\
\hline Male & 68 \\
\hline Female & 100 \\
\hline \multicolumn{2}{|l|}{ Race } \\
\hline Caucasian & 53 \\
\hline African-American & 50 \\
\hline Hispanic & 34 \\
\hline Asian & 28 \\
\hline Other & 3 \\
\hline \multicolumn{2}{|l|}{ Indications } \\
\hline CBD stone (known/suspected) & 54 \\
\hline Pancreatic mass/cyst & 27 \\
\hline CBD stent/stricture/bile leak & 20 \\
\hline Chronic pancreatitis & 18 \\
\hline Abnormal liver enzymes/RUQ pain & 18 \\
\hline Dilated PD/CBD & 14 \\
\hline Cholangitis & 8 \\
\hline Gallbladder mass & 4 \\
\hline Other mass & 4 \\
\hline Abnormal lymphadenopathy & 3 \\
\hline FAP & 1 \\
\hline Celiac plexus neurolysis & 1 \\
\hline
\end{tabular}

CBD, common bile duct; RUQ, right upper quadrant; PD, pancreatic duct; FAP, familial adenomatous polyposis. found in 30/168 patients (18\%). $\odot$ Table 3 lists the specific details. EGD with a gastroscope after a linear EUS resulted in additional lesion findings in $17 / 83$ patients $\left(20.5 \%, X^{2}=13.385, P=0.00025\right)$. EGD with a gastroscope after an ERCP with a duodenoscope resulted in additional lesions findings in $10 / 52$ patients $\left(19.2 \%, X^{2}\right.$ $=9.987, P=0.00157)$. EGD with a gastroscope after the use of both a linear echoendoscope and a duodenoscope resulted in additional lesions findings in $3 / 33$ patients $\left(9 \%, X^{2}=3.219, P=0.07\right)$. Additional lesions diagnosed with a forward-viewing gastroscope and missed with a side-viewing duodenoscope included Barrett's esophagus (2), esophageal candidiasis (1), esophageal varices (1), reflux esophagitis (1), focal $H$. pylori-positive gastritis (2), and gastric ulcers in the antrum (2) and fundus (1). Additional lesions missed with an oblique-viewing linear echoendoscope included Barrett's esophagus (2), esophageal candidiasis (1), esophageal varices (2), reflux esophagitis (1), esophageal ulcer (1), focal H. pylori gastritis (5), gastric ulcer in the antrum (1), gastric adenoma with high-grade dysplasia in the fundus (1), duodenal angioectasia (second part) (1), and adenomatous duodenal polyps (second part) (2). Focal H. pylori gastritis was seen as focal erythema that histology from biopsies showed H. pylori. Finally, lesions missed by both non-forward-viewing endoscopes in a single procedure included Barrett's esophagus (2) and esophageal varices (1) ( $\bullet$ Table 3 ). Two lesions, a duodenal polyp and an ampullary adenoma, were visualized with a side-viewing duodenoscope and not seen with the standard gastroscope. No upper gastrointestinal malignancies were diagnosed. No adverse events were reported in any of the patients undergoing standard EGD following a EUS or ERCP.

Table 3 Characteristics of the pathology found organized by type of management change and endoscope that diagnosed the lesion.

\begin{tabular}{|c|c|c|c|c|}
\hline & $\operatorname{ERCP}(n=52)$ & Linear EUS $(n=83)$ & ERCP \& EUS $(n=33)$ & Total $(n=168)$ \\
\hline \multicolumn{5}{|l|}{$\begin{array}{l}\text { Additional Findings on EGD } \\
\text { Prompting Change in }\end{array}$} \\
\hline Management & $10(P=0.00157)$ & $17(P=0.00025)$ & $3(P=0.07)$ & 30 \\
\hline Medication changes & 9 & 11 & 2 & 22 \\
\hline \multirow[t]{2}{*}{ Esophageal } & 4 & 5 & 2 & 11 \\
\hline & $\begin{array}{l}\text { Barrett's (2) } \\
\text { Candida esophagitis ( } 1 \text { ) } \\
\text { Reflux esophagitis ( } 1 \text { ) }\end{array}$ & $\begin{array}{l}\text { Barrett's (2) } \\
\text { Candida esophagitis ( } 1 \text { ) } \\
\text { Reflux esophagitis (1) } \\
\text { Ulcer (1) }\end{array}$ & Barrett's $(2)^{1}$ & \\
\hline \multirow[t]{2}{*}{ Gastric } & 5 & 6 & 0 & 11 \\
\hline & $\begin{array}{l}\text { Antral gastric ulcer (2) } \\
\text { Fundic gastric ulcer (1) } \\
\text { Focal H.pylori gastritis (2) }\end{array}$ & $\begin{array}{l}\text { Antral gastric ulcer (1) } \\
\text { Focal H.pylori gastritis (5) }\end{array}$ & & \\
\hline Duodenal & 0 & 0 & 0 & 0 \\
\hline $\begin{array}{l}\text { Endoscopic treatment or } \\
\text { additional procedures }\end{array}$ & 3 & 8 & 3 & 14 \\
\hline \multirow[t]{2}{*}{ Esophageal } & 3 & 4 & 3 & 10 \\
\hline & $\begin{array}{l}\text { Barrett's }(2)^{*} \\
\text { Varices }(1)\end{array}$ & $\begin{array}{l}\text { Barrett's (2)* } \\
\text { Varices (2) }\end{array}$ & $\begin{array}{l}\text { Barrett's (2)* } \\
\text { Varices (1) }\end{array}$ & \\
\hline \multirow[t]{2}{*}{ Gastric } & 0 & 1 & 0 & 1 \\
\hline & & Fundic adenoma with HGD (1) & & \\
\hline \multirow[t]{2}{*}{ Duodenal } & 0 & 3 & 0 & 3 \\
\hline & & $\begin{array}{l}\text { Angioectasia in D2 (1) } \\
\text { Adenomatous polyp in D2 (2) }\end{array}$ & & \\
\hline
\end{tabular}

HGD, high-grade dysplasia

* Both medication and procedural changes 


\section{Discussion}

$\nabla$

ERCP and EUS are increasingly utilized in pancreaticobiliary exams. While these endoscopes are required to traverse the upper gastrointestinal tract to obtain their images, it is unclear if the endoscopist should be performing a detailed exam of the upper gastrointestinal tract during the same procedure. Our study helps answer this question as the literature to our knowledge is surprisingly sparse on this topic. In our study we found that significant incidental upper gastrointestinal lesions is found when a formal exam is performed with a gastroscope.

A recent study of 204 patients found that $22 \%$ were found to have clinically meaningful lesions on EGD prior to EUS for pancreaticobiliary indications and the authors of the study supported performing an EGD for any patient undergoing EUS [3]. However, in the study, an EGD was performed before the EUS exam, and therefore it is not known if the clinically meaningful lesions would have been detected by an oblique-viewing echoendoscope without performing an EGD. In our study, an EGD was performed after a linear EUS exam and resulted in additional lesions findings in $17 / 83$ patients (20.5\%). Furthermore, no studies have evaluated the role of an EGD in detecting clinically meaningful lesions when performed either before or after an ERCP. In our study, an EGD with a gastroscope after an ERCP with a duodenoscope resulted in additional lesions findings in 10/52 patients (19.2\%).

A previous study of 172 patients compared EGD and endoscopic ultrasound with radial EUS for the evaluation of upper abdominal pain and demonstrated combined luminal findings in $25 \%$ of the patients [4]. Patients were randomized to undergo luminal examination with both a standard gastroscope and an obliqueviewing radial echoendoscope by separate gastroenterologists in tandem prior to sonographic examination. In the subset analysis, there were no overall differences between the two scopes for detecting mucosal lesions, but the radial echoendoscope missed several Barrett's esophagus, gastric ulcers, and submucosal lesions. The ability to take biopsies with the radial scope was also limited, requiring a 56\% conversion rate to a standard endoscope. However, this study cohort only included patients who were referred to gastroenterologists for diagnostic EGDs to evaluate their upper abdominal pain, therefore, the findings cannot be extrapolated to asymptomatic patients and patients referred for EUS for other indications.

Another study of 200 patients with dyspepsia demonstrated that oblique-viewing radial EUS had sensitivity and specificity of $80 \%$ and $95 \%$, respectively, for detecting luminal lesions when compared to EGD [5]. In this study, patients who were referred for dyspepsia underwent radial EUS followed by EGD to look for both luminal and extraluminal etiologies of their symptoms. Luminal lesions that were missed by EUS included duodenal ulcers and reflux esophagitis. Given that the primary goal of this study was to evaluate an EUS-based management strategy for dyspepsia, only findings that accounted for dyspeptic symptoms were reported. This study cohort included only patients with dyspepsia. Because clinically significant lesions do not always cause symptoms, these findings do not translate to our study population who were undergoing EUS for both symptomatic and asymptomatic indications.

Our study evaluated patients referred for ERCP and/or EUS.Although it is not known how many patients had undergone previous upper endoscopic evaluation prior to the procedures analyzed, our findings demonstrate the importance of performing an EGD during ERCP and/or EUS. Our results among 168 patients showed that EGD with a forward-viewing gastroscope yielded additional meaningful findings in 19\% (10/52) of patients following an ERCP with a duodenoscope, 20\% (17/83) of patients following a linear EUS, and $9 \%$ (3/33) of patients utilizing both a duodenoscope and a linear echoendoscope. Our findings coincide with previous published rates of incidental lesions requiring management changes found during EGD performed for various reasons, which range from $22 \%$ to $62 \%$, but it is the first to show the high miss rate of significant lesions with both duodenoscopes and linear EUS echoendoscopes [3,6,7].

Prior to data analysis, we hypothesized that nearly all of the missed lesions during non-forward-viewing endoscopy would be esophageal given the semi-"blind" passage of the scope through the narrowed tubular lumen of the esophagus compared to the stomach and duodenum. However, 15/30 of the missed lesions (50\%) were found in the stomach and duodenum, suggesting that luminal diameter was not the main factor contributing to missed lesions.

Additional lesions diagnosed by EGD requiring endoscopic treatment included argon plasma coagulation (APC) of an angioectasia, endoscopic mucosal resection of a gastric adenoma with high-grade dysplasia, and variceal band ligation. Additional lesions requiring further endoscopic surveillance included Barrett's esophagus, esophageal varices, and gastric ulcers. Additional significant medical interventions included treatment for $H$. $p y$ lori, esophageal varices, and cirrhosis workup and management. One patient with a pancreatic head cyst causing partial duodenal obstruction diagnosed on forward-viewing endoscopy was admitted to the hospital for management of her symptoms.

In this study, we performed the EGD after the indicated ERCP or EUS procedure to maximize time for the main procedure in the event that the procedure had to be terminated prematurely. Previous data have suggested that performing standard upper endoscopy prior to EUS may detect luminal lesions that will affect subsequent EUS in $9.8 \%$ to $12 \%$ of patients, thus preventing potential complications during EUS $[3,8]$. However, other studies have shown EUS to be a safe procedure with a low complication rate even if performed without a prior EGD [9]. In our study, zero adverse events/complications were experienced during non-forward-viewing exams. It should be noted that these patients had no upper gastrointestinal symptoms. Patients with upper gastrointestinal symptoms underwent EGD first and were not included in this study.

This study strongly supports the notion that standard forwardviewing endoscopy should be performed at the same session of pancreaticobiliary EUS and ERCP. This study did not evaluate the increased cost of performing a standard EGD. The previously described study on this topic did not evaluate cost effectiveness either [3]. The additional costs would be significant as there are costs associated with cleaning the additional gastroscope, equipment to biopsy (e.g., biopsy forceps), provide additional therapy (e.g., APC of lesions), physician fee for the additional procedure, etc. However, the additional lesions discovered in this cohort were significant and would likely be clinically significant in the future, adding to the cost of future gastrointestinal healthcare (e.g., cost of gastrointestinal bleeding in incidentally found vascular ectasias or varices, gastric cancer in the incidental gastric adenoma with high-grade dysplasia, duodenal cancer in the incidental duodenal adenoma, etc). It is also possible that some of the incidentally found lesions increased healthcare costs without clinical benefit (e.g., enrolling patients with incidentally found non-dysplastic Barrett's esophagus that will not progress to neo- 
plasia in a surveillance program, eradication of $H$. pylori that never would have caused significant disease, etc.). Future studies are needed to investigate the cost effectiveness of forward-viewing endoscopy at the time of pancreaticobiliary EUS and ERCP. This study also shows that in patients without upper gastrointestinal symptoms who undergo pancreaticobiliary EUS or ERCP, the order of forward-viewing endoscopic exam is irrelevant. There were no complications associated with the forward-viewing endoscopy when performed after EUS or ERCP. There is a theoretical risk that an oblique or side-viewing scope which traverses an unknown esophageal or duodenal stricture/lesion could possibly lead to a perforation. For this theoretical risk only, we would recommend that the forward-viewing endoscopy be performed before every pancreaticobiliary EUS and ERCP.

This study has certain limitations which should be noted, including the limitations inherent in its retrospective design. However, all endoscopic findings for each exam were recorded separately on the procedure report, thus the groups were not difficult to compare. Another limitation is that this study only included one endoscopist's procedures. Other practicing therapeutic endoscopists at our institution were not included in the study because their standard of care was to perform an EGD prior to the EUS or ERCP. In addition, an inherent limitation for which we cannot account is the theoretical subconscious decreased effort of the endoscopist to look for incidental upper gastrointestinal tract lesions knowing that a forward-viewing endoscopy is going to be performed. However, a significant number of lesions were discovered on EUS and ERCP before forward-viewing endoscopy, which makes this less likely.

In summary, the current study demonstrates that non-forwardviewing duodenoscopes and linear echoendoscopes miss a significant number of meaningful incidental upper gastrointestinal luminal lesions. Performing an upper endoscopy with a forwardviewing gastroscope at the time of ERCP or EUS leads to an in- creased yield of meaningful incidental upper gastrointestinal lesions. Gastroenterologists who perform linear EUS or ERCP should strongly consider performing a formal EGD with a gastroscope at the time of the EUS or ERCP exam. Further prospective studies are warranted in order to make clinical practice recommendations.

\section{Competing interests: None}

\section{References}

1 Andriulli A, Loperfido S, Napolitano $G$ et al. Incidence rates of post ERCP complications. Am J Gastroenterol 2007; 102: 781 - 1788

2 Mekky MA, Abbas WA. Endoscopic ultrasound in Gastroenterology: From diagnosis to therapeutic implications. World J Gastroenterol 2014; 20: $7801-7807$

3 Sahakian AB, Aslanian AS, Mehra M et al. The utility of esophagogastroduodenoscopy before endoscopic ultrasonography in patients undergoing endoscopic ultrasonography for pancreatio-biliary and mediastinal indications. J Clin Gastroenterol 2013; 47: 857-860

4 Chang KJ, Erickson RA, Chak A et al. EUS compared with endoscopy plus transabdominal US in the initial diagnostic evaluation of patients with upper abdominal pain. Gastrointest Endosc 2010; 72: 967-974

5 Lee YT, Lai AC, Hui $Y$ et al. EUS in the management of uninvestigated dyspepsia. Gastrointet Endosc 2002; 56: 842 - 848

6 Knopp $H$, Halm $U$, Lamberts $R$ et al. Incidental and ablation-induced findings during upper gastrointestinal endoscopy in patients after ablation of atrial fibrillation: A retrospective study of 425 patients. Heart Rhythm 2014; 11: 574-578

7 Sharaf RN, Weinshel EH, Bini EJ et al. Endoscopy plays an important preoperative role in bariatric surgery. Obesity Surgery 2004; 14: $1367-1372$

8 El-Dika S, Baltz J, White GE et al. Leading the blind: standard upper endoscopy provides an important road map prior to endoscopic ultrasound in patients without known luminal pathology. Gastrointest Endosc 2009; 69: AB336

9 ASGE Standards of Practice Committee. Early DS, Acosta RD et al. Adverse events associated with EUS and EUS with FNA. Gastrointest Endosc 2013; 77: 839-843 